\title{
SUMMABILITY-PRESERVING FUNCTIONS
}

\author{
EDWARD C. POSNER
}

The questions answered in this paper suggest themselves naturally. The first lemma is a consequence of a result of R. C. Buck.

Lemma. Let $f$ be a function of a complex variable and $A$ a Toeplitz matrix such that $\left\{f\left(x_{n}\right)\right\}$ is $A$-summable whenever $\left\{x_{n}\right\}$ converges. Then $f$ is continuous.

PRoof. Every subsequence of $\left\{x_{n}\right\}$ converges, so that every subsequence of $\left\{f\left(x_{n}\right)\right\}$ is $A$-summable, by hypothesis. By [1], applied to complex sequences, $\left\{f\left(x_{n}\right)\right\}$ is actually convergent. (For the proof of [1] can be modified to apply to complex sequences. Or, see [2, Theorem 2], for a proof in a slightly more general context.) Let $\left\{x_{n}\right\}$ converge to $x$. The sequence $x_{1}, x, x_{2}, x, \cdots$ is also convergent, so that the sequence $f\left(x_{1}\right), f(x), f\left(x_{2}\right), f(x), \cdots$ converges. This shows that $\left\{f\left(x_{n}\right)\right\}$ converges to $f(x)$ whenever $\left\{x_{n}\right\}$ converges to $x$. Thus the continuity of $f$ is proved.

The converse of the lemma is a consequence of the definition of Toeplitz matrix. The lemma is of course also true for real-valued functions of a real variable. The same comment applies also after the following theorem.

Theorem. Let $A=\left(a_{i n}\right), 1 \leqq i, n<\infty$, be a Toeplitz matrix, and $f$ a function of a complex variable, such that if $\left\{x_{n}\right\}$ is a bounded $(C, 1)$ summable sequence, then $\left\{f\left(x_{n}\right)\right\}$ is A-summable. Then $f$ is linear. If in addition $f$ is not the constant function, then $A$ sums every bounded $(C, 1)$-summable sequence to its $(C, 1)$ sum.

Proof. Every convergent sequence is a bounded $(C, 1)$-summable sequence. Thus $f$ satisfies the hypotheses of the lemma. Hence $f$ is continuous. We will prove that $f((a+b) / 2)=(f(a)+f(b)) / 2$ for all $a$ and $b$. It is well known that a continuous function with this property is linear.

Consider the sequence $a, b, a, b, \cdots$, which is a bounded $(C, 1)$ summable sequence. In fact, we may interpolate any number of terms $(a+b) / 2$ between successive terms of this sequence, infinitely many if we wish, and still have a bounded $(C, 1)$-summable sequence. To prove this, let us examine the average of the first $n$ terms of such an interpolated sequence. These first $n$ terms consist of $r b$ 's, $r$ or $r+1 a$ 's,

Presented to the Society, April 19, 1960 under the title Two theorems on $(C, 1)$ summability; received by the editors December 19, 1958 and, in revised form, December 7, 1959 and February 12, 1960. 
and $n-2 r$ or $n-2 r-1((a+b) / 2)$ 's, say. The average of these $n$ terms is therefore equal to $(1 / n)(r(a+b)+(n-2 r)(a+b) / 2)$ or $(1 / n)(r(a+b)+a+(n-2 r-1)(a+b) / 2)$, that is, to $(a+b) / 2$ or $((a+b) / 2)(1-1 / n)+a / n$. As $n$ approaches infinity, the Cesaro means of such an interpolated sequence therefore converge to $(a+b) / 2$. This proves the assertion. By the hypothesis of this theorem, we conclude that any sequence $f(a), f(b), f(a), f(b), \cdots$, with any number of $f((a+b) / 2)$ 's interpolated into it is $A$-summable. We shall use this fact shortly.

If $f$ is the constant function, there is nothing more to prove. Throughout the remainder of the proof, $f$ will be nonconstant. Let $a, b$ be such that $f(a)=p, f(b)=q$, with $p \neq q$. The sequence $p, q$, $p, q, \cdots$ is $A$-summable by hypothesis. Then the sequence $p-q, 0$, $p-q, 0, \cdots$ is also $A$-summable. For this sequence is obtained from the preceding sequence by subtracting the sequence $q, q, q, \cdots$. Upon dividing each term of $p-q, 0, p-q, 0, \cdots$ by $p-q$, we conclude that the sequence $1,0,1,0, \ldots$ is $A$-summable. In other words, $\lim _{i \rightarrow \infty} \sum_{n \text { odd }} a_{i n}$ exists; call it $r$. Since for every Toeplitz matrix, $\lim _{i \rightarrow \infty} \sum_{n=1}^{\infty} a_{i n}=1$, we must have $\lim _{i \rightarrow \infty} \sum_{n \text { even }} a_{i n}=1-r$.

Now consider any sequence $f(a), f(b), f(a), f(b), \cdots$. We shall interpolate $f((a+b) / 2)$ 's into this sequence in such a way that we obtain a new sequence, which we know to be $A$-summable by the above argument, and yet which has a subsequence of its sequence of auxiliary means under $A$ convergent to $f((a+b) / 2)$, and another subsequence of its sequence of auxiliary means under $A$ convergent to $r f(a)+(1-r) f(b)$. But since the sequence of auxiliary means under $A$ of the interpolated sequence converges, and since a convergent sequence has but one limit point, we must conclude that these two limit points coincide.

Let $M$ be the maximum of the absolute values of the three numbers $f(a), f(b), f((a+b) / 2)$. Let $N_{1}$ be even and so large that $\sum_{n>N_{1}}\left|a_{1 n}\right|$ $<1 / 2 M$. Then for any sequence $\left\{c_{n}\right\}$ composed only of terms chosen from $f(a), f(b), f((a+b) / 2)$, whose first $N_{1}$ terms are the first $N_{1}$ terms of the sequence $f(a), f(b), f(a), f(b), \cdots$, we observe that $\sum_{n=1}^{\infty} a_{i n} c_{n}$ differs in absolute value from $f(a) \sum_{n \text { odd }} a_{1 n}+f(b) \sum_{n \text { even }} a_{1 n}$ by less than $2 M \cdot 1 / 2 M=1$. Let $i_{1}=1$ and choose $i_{2}>i_{1}$ and so large that $\sum_{n=1}^{N_{1}}\left|a_{i_{2}, n}\right|<1 / 2 M$. We now start interpolating terms $f((a+b) / 2)$. Let $N_{2}$ be even, $>N_{1}$, and so large that $\sum_{n>N_{2}}\left|a_{i_{2}, n}\right|<1 / 4 M$. Then any sequence $\left\{c_{n}\right\}$ composed only of terms $f(a), f(b), f((a+b) / 2)$, whose terms from $N_{1}+1$ up to $N_{2}$ are all equal to $f((a+b) / 2)$, has the property that $\sum_{n=1}^{\infty} a_{i_{2}, n} c_{n}$ differs in absolute value from $f((a+b) / 2) \sum_{n=1}^{\infty} a_{i_{2}, n}$ by less than $M \cdot 1 / 2 M+2 M \cdot 1 / 4 M=1$. We shall 
now find $i_{3}>i_{2}$ and so large that $\sum_{n=1}^{N_{2}}\left|a_{i_{3}, n}\right|<1 / 4 M$. Now choose $N_{3}$ even, $>N_{2}$, and so large that $\sum_{n>N_{3}}\left|a_{i_{3}, n}\right|<1 / 8 M$. We now leave in our sequence $\left\{c_{n}\right\}$ that we are constructing, terms $a, b, a$, $b, \cdots$ starting from $N_{2}+1$ and stopping at $N_{3}$. Any sequence $\left\{c_{n}\right\}$ with such terms in the indicated positions and its remaining terms chosen from among $f(a), f(b), f((a+b) / 2)$, has the property that $\sum_{n=1}^{\infty} a_{i_{3}, n} c_{n}$ differs in absolute value from $f(a) \sum_{n}$ odd $a_{i_{3}, n}$ $+f(b) \sum_{n \text { even }} a_{i_{3}, n}$ by less than $M \cdot 1 / 4 M+2 M \cdot 1 / 8 M=1 / 2$. Choose $i_{4}>i_{3}$ and so large that $\sum_{n=1}^{N_{3}}\left|a_{i_{4}, n}\right|<1 / 4 M$. Then choose $N_{4}$ even, $>N_{3}$, and so large that $\sum_{n>N_{4}}\left|a_{i_{4}, n}\right|<1 / 8 M$. Now interpolate $f((a+b) / 2)$ from the $N_{3}+1$ to the $N_{4}$ position. Any sequence $\left\{c_{n}\right\}$ with $f((a+b) / 2)$ in these positions and its remaining terms chosen from among $f(a), f(b), f((a+b) / 2)$, has the property that $\sum_{n=1}^{\infty} a_{i_{4}, n} c_{n}$ differs in absolute value from $f((a+b) / 2) \sum_{n=1}^{\infty} a_{i_{4}, n}$ by less than $M \cdot 1 / 4 M+2 M \cdot 1 / 8 M=1 / 2$. Continuing in this fashion, we finally obtain a sequence $\left\{c_{n}\right\}$ with the property that

$$
\begin{aligned}
\left|\sum_{n=1}^{\infty} a_{i_{2 k-1}, n} c_{n}-f(a) \sum_{n \text { odd }} a_{i_{2 k-1}, n}-f(b) \sum_{n \text { even }} a_{i_{2 k-1}, n}\right| & <\frac{1}{k}, \\
k & =1,2, \cdots,
\end{aligned}
$$

and

$$
\left|\sum_{n=1}^{\infty} a_{i_{2 k}, n} c_{n}-f\left(\frac{a+b}{2}\right) \sum_{n=1}^{\infty} a_{i_{2 k}, n}\right|<\frac{1}{k}, \quad k=1,2, \cdots .
$$

Given any $\epsilon>0$, if $k>2 / \epsilon$ and also so large that

$$
\begin{gathered}
\left|\sum_{n \text { odd }} a_{i_{2 k-1}, n}-r\right|<\frac{\epsilon}{4 M}, \quad\left|\sum_{n \text { even }} a_{i_{2 k-1}, n}-(1-r)\right|<\frac{\epsilon}{4 M}, \\
\left|\sum_{n=1}^{\infty} a_{i_{2 k}, n}-1\right|<\frac{\epsilon}{2 M},
\end{gathered}
$$

then we have

$$
\begin{gathered}
\left|\sum_{n=1}^{\infty} a_{i_{2 k-1}, n} c_{n}-r f(a)-(1-r) f(b)\right|<\frac{\epsilon}{2}+\frac{\epsilon}{4}+\frac{\epsilon}{4}=\epsilon, \\
\left|\sum_{n=1}^{\infty} a_{i_{2 k}, n} c_{n}-f\left(\frac{a+b}{2}\right)\right|<\frac{\epsilon}{2}+\frac{\epsilon}{2}=\epsilon .
\end{gathered}
$$

From this, we conclude that the subsequence of the auxiliary means of $\left\{c_{n}\right\}$ corresponding to those rows of $A$ indexed by $i_{2 k-1}, k=1,2, \cdots$, converges to $r f(a)+(1-r) f(b)$. Similarly, the subsequence of the auxiliary means of $\left\{c_{n}\right\}$ under $A$ corresponding to those rows of $A$ 
indexed by $i_{2 k}, k=1,2, \cdots$, converges to $f((a+b) / 2)$. Since a convergent sequence has but one limit point, we conclude that $f((a+b) / 2)$ $=r f(a)+(1-r) f(b)$. Starting with the sequence $b, a, b, a, \cdots$, we likewise conclude that $f((b+a) / 2)=r f(b)+(1-r) f(a)$. Thus for all $a$ and $b, r f(a)+(1-r) f(b)=r f(b)+(1-r) f(a)$. Since $f$ is nonconstant, we can choose $a, b$ such that $f(a) \neq f(b)$. Then $r(f(a)-f(b))$ $=(1-r)(f(a)-f(b))$. Thus $r=1-r$, or $r=1 / 2$. Then $f((a+b) / 2)$ $=(1 / 2) f(a)+(1-1 / 2) f(b)$, that is, $f((a+b) / 2)=(f(a)+f(b)) / 2$ for all $a$ and $b$. Since $f$ is continuous, we conclude that $f$ is linear.

To prove the last part of the theorem, let $f(z)=c z+d$ with $c \neq 0$. The hypothesis of the theorem tells us that $\left\{c x_{n}+d\right\}$ is $A$-summable whenever $\left\{x_{n}\right\}$ is a bounded $(C, 1)$-summable sequence. Subtracting the sequence $d, d, d, \ldots$ from this sequence and dividing by $c$, we find that $\left\{x_{n}\right\}$ is $A$-summable whenever $\left\{x_{n}\right\}$ is a bounded $(C, 1)$ summable sequence. Theorem 1 of [3] is now exactly what one needs to conclude that $A$ sums every bounded $(C, 1)$-summable sequence to its $(C, 1)$ sum. This concludes the proof of the theorem.

The theorem is of course false for summability methods (as opposed to $(C, 1))$ whose convergence field is too small.

A question which arises in connection with the theorem has been answered by Professor H. Hanani: Namely, if the Toeplitz matrix $A$ sums every bounded $(C, 1)$-summable sequence, does it sum every $(C, 1)$-summable sequence? The answer is "no." For let $a_{i n}=1 / i$, $1 \leqq n \leqq i, a_{i, i^{2}}=1 / i, a_{i n}=0$ otherwise. The matrix $A=\left(a_{i n}\right)$ is a Toeplitz matrix which sums every bounded $(C, 1)$-summable sequence, as is easy to see. But the sequence $\left\{(-1)^{n+1} n^{1 / 2}\right\}, n=1,2, \cdots$, is $(C, 1)$-summable (to zero), whereas the sequence of its odd auxiliary means under $A$ converges to +1 , its even ones to -1 . Another question whose answer is "no" is this: if the Toeplitz matrix $A$ sums every bounded $(C, 1)$-summable sequence, does it give the $(C, 1)$ sum for any $(C, 1)$-summable sequence which it happens to sum? For let $A=\left(a_{i n}\right)$ with $a_{i n}=1 / i, 1 \leqq n \leqq i, a_{i, i^{2}}=(-1)^{i+1} \cdot 1 / i, a_{i n}=0$ otherwise. Then the same sequence as used above is summable by this matrix to 1 , not zero.

\section{BIBLIOGRAPHY}

1. R. C. Buck, An addendum to "A note on subsequences," Proc. Amer. Math. Soc. vol. 7 (1956) pp. 1074-1075.

2. E. C. Posner, Accumulability and continuous functions, Duke Math. J., to appear.

3. A. L. Brudno, Summation of bounded sequences by matrices, Mat. Sb. N.S. vol. 16 (58) (1945) pp. 191-247.

UNIVERSITY OF WISCONSIN 